\title{
DETERMINANTS OF NEONATAL MORTALITY IN VILLAGES OF BALLABGARH BLOCK, HARYANA: A RETROSPECTIVE POPULATION BASED STUDY USING HIERARCHICAL MODEL
}

\section{R. Upadhyay}

Centre for Community Medicine, All India Institute of Medical Sciences, New Delhi, India

Aim: To identify the determinants of neonatal mortality to support strategy development to reduce neonatal mortality rate in the study area as well as on a national level.

Methods: A nested case-control study involving villages under the Field Practice Area of Comprehensive Rural Health Services Project (CRHSP), Ballabgarh, Haryana serving a population of 85522. The study period was from 2005 to 2009. The data were obtained from Health Management Information System (HMIS) and analysed using multivariate logistic regression analysis. A hierarchical approach was used to analyze the factors associated with neonatal deaths, using community level factors, socio-economic status and biological determinants.

Results: The total live births during the study period were 10392 and neonatal deaths were 248. Socioeconomic determinants (Low educational status of parents [OR 2.1; 95\% CI, 1.4-3.3]; father's occupation [OR 1.8; 95\% CI, 1.0-3.0]; Rajput caste [OR 2.0; 95\% CI 1.2-3.4] were responsible for a major fraction $(46 \%)$ of neonatal deaths. Community level factors (villages with no health facility [OR $1.5 ; 95 \%$ CI, 1.0 2.1]; villages with population $>6000$ [OR $1.7 ; 95 \%$ CI, 1.2-2.5]) explained a total of $28 \%$ of all neonatal deaths. Proximate determinants (early childbearing age of mother ( $<20$ years) [OR 2.0, 95\% CI 1.2-3.2]) were least important. All the three level of variables seemed to act independently with little mediation among them.

Conclusion: A comprehensive strategy addressing community level, socio-economic and biological determinants is required to reduce neonatal mortality. 